\title{
Iron oxide nanoparticles and the mechanisms of immune recognition of nanomedicines
}

\author{
"Superparamagnetic iron oxides are a nice illustration of how \\ understanding the mechanisms of immune recognition could \\ be useful to decrease immune uptake."
}

First draft submitted: 4 January 2016; Accepted for publication: 8 February 2016; Published online: 16 March 2016

\section{Keywords: blood $\bullet$ complement $\bullet$ leukocytes $\bullet$ macrophages $\bullet$ nanoparticles $\bullet$ scavenger receptors}

Nanomaterials are foreign substances that are treated by the immune system just like foreign invading pathogens. The immune system (innate immunity for the most part) is trained to recognize regular arrangements of chemical groups, which is what engineered nanomaterials are. The immune recognition poses multiple challenges to the use of nanomaterials for drug and gene delivery. One of the untoward results of immune recognition to nanomaterials once they are injected into the circulation is activation of the complement cascade. The complement system is a central component of the innate immunity accounting for about $5 \%$ of globulins in serum and is responsible for recognizing, eliminating and destroying pathogens [1]. Uncontrolled complement activation is known to cause inflammation, immune cell activation, increased vascular permeability and even tumor growth [1-3]. Nanocarriers can cause complement activation-related pseudoallergy or CARPA (term introduced by Dr Szebeni [4]). CARPA is a known phenomenon following infusion of Doxil (liposomal doxorubicin), Taxol (cremophorpaclitaxel), Feridex (dextran iron oxide) and SandImmune (cremophor-cyclosporine A). Numerous reports demonstrated complement activation by carbon nanotubes [5], micelles [6], liposomes, [7] polymer [8] and gold nanoparticles [9] in vitro and in vivo. Additional effects of complement activation are nanoparticle opsonization with complement fragments and subsequent clearance by macrophages and leukocytes. Besides complement, other mechanisms of recognition and clearance of nanoparticles are known. Scavenger receptors (SRs) are professional phagocytic pattern recognition receptors that were discovered in 1979 [10] and are responsible for opsonin-independent binding and uptake of oxidized low-density lipoprotein (oxLDL), pathogens, environmental particles and apoptotic cells. The universal feature of SRs is that their ligands possess a pattern of negative charges. SRs are implicated in the immune uptake of numerous nanoparticle types [11].

The main criticism of studies of immune mechanisms of clearance of nanomedicines in order to prolong circulation and decrease complement activation could be summarized in two questions: why not make thousands of nanoparticulate compounds (by combinatorial approach) and one of them will work? How the immune recognition can be blocked after learning that proteins (receptors) X, Y and $\mathrm{Z}$ mediate nanoparticle clearance? The answer to these questions is complex. Can we use the rational knowledge to modify nanoparticle structure to avoid the immune receptors? Superparamagnetic iron oxides (SPIO) are a nice illustration of how understanding the mechanisms of immune recognition could be useful to decrease immune

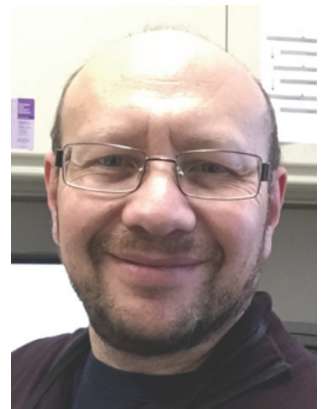

Dmitri Simberg

The Skaggs School of Pharmacy \& Pharmaceutical Sciences, Department of Pharmaceutical Sciences, University of Colorado Anschutz Medical Campus, 12850 East Montview Blvd., Aurora, CO 80045, USA

Tel.: +1 3037248241

dmitri.simberg@ucdenver.edu

Future
Medicine
part of 
uptake. SPIO are nanoparticle-based contrast magnetic resonance imaging (MRI) agents that result in a negative contrast in images. SPIO comprise multiple magnetite-maghemite $\left(\mathrm{Fe}_{3} \mathrm{O}_{4}\right.$ and $\left.-\mathrm{Fe}_{2} \mathrm{O}_{3}\right)$ crystals. The surface of the crystals is anionic. Biopolymer coating (dextran, carboxydextran, chitosan, etc.) is necessary to impart water solubility and colloidal stability to the particles. The main translational disadvantages of these nanoparticles are immune-related adverse effects and high nonspecific uptake by following injection. SPIOs show rapid clearance and uptake by liver and spleen macrophages [12] and leukocytes [13]. Several dextrancoated SPIO formulations showed severe anaphylacticlike reactions in patients (Feridex, Combidex). Despite being withdrawn from the market, dextran coated SPIO can be utilized to learn about mechanisms of interactions between nanoparticles and the body milieu. SPIO is an excellent example of a nanomaterial with pathogen-like properties. Iron oxide crystal lattice exhibits a molecular pattern of periodical anionic charges, whereas dextran is the biopolymer composed of periodic sugar groups. Dextran maintains aqueous solubility of nanoparticles, but it does not cover the crystal surface and the anionic crystals are significantly exposed [12,14]. Anionic charge patterns on the surface of iron oxide could be recognizable by scavenger receptors [15]. Hence, sterically blocking access to the anionic iron oxide core, for example by using longer dextran or forming hydrogel prevents recognition by scavenger receptors [16], including main macrophage receptors SR-AI/II. Complement activation is a more complex issue. In mice, dextran triggers complement activation of SPIO via the lectin (sugar recognition) pathway [17]. Dextran crosslinking with epichlorohydrin alters the sugar structure and blocks complement activation in mice [18]. At the same time, dextran is immunogenic and some individuals have anti-dextran antibodies that can trigger complement via the classical pathway [19]. Moreover, complement activity toward nanosurfaces is higher in humans than in other species [20]. The main reason for the difference is the enhanced alternative pathway activity in human serum. Therefore, blocking lectin and the classical pathway by crosslinking did not prevent complement activation in humans (although it

\section{References}

1 Ricklin D, Hajishengallis G, Yang K, Lambris JD. Complement: a key system for immune surveillance and homeostasis. Nat. Immunol. 11(9), 785-797 (2010).

2 Moghimi SM. Cancer nanomedicine and the complement system activation paradigm: anaphylaxis and tumour growth. J. Control. Release 190, 556-562 (2014). decreased somehow the leukocyte uptake due to formation of hydrogel of nanoparticle surface) [13]. Over the past several years the alternative pathway has been shown to be the essential pathway of complement activation in health and disease, and for many types of drug delivery nanoplatforms, including liposomes and PLGA-lipid nanoparticles, it has been shown to be a critical pathway for complement activation. Understanding the mechanisms of assembly of the alternative pathway on SPIO could be instrumental in developing novel strategies to block C3 opsonization and complement activation on nanosurfaces.

\section{"SPIO is an excellent example of a nanomaterial with pathogen-like properties.'}

It must be reminded that complement and scavenger receptors are not the only mechanism to intercept nanoparticles by macrophages. In that regard, SPIO can be used to further understand clearance mechanisms and receptors engaged in nanosurface recognition. Due to being magnetic, the nanoparticles can be separated over a standard magnetic column and further analyzed by proteomics approaches for multiple redundant receptors responsible for clearance of SPIOs by immune cells. For these studies, SPIO have unique advantages over 'classical' pathogens due to 'built-in' magnetic properties and controllable surface modifications, which could enable efficient capturing, purification and characterization of redundant receptors.

In conclusion, we suggest that understanding the mechanisms of interaction between immune system and SPIO can promote the development of materials with improved hemocompatibility, pharmacokinetic properties and safety profile.

\section{Financial \& competing interests disclosure}

The author has no relevant affiliations or financial involvement with any organization or entity with a financial interest in or financial conflict with the subject matter or materials discussed in the manuscript. This includes employment, consultancies, honoraria, stock ownership or options, expert testimony, grants or patents received or pending, or royalties.

No writing assistance was utilized in the production of this manuscript.

3 Volk LD, Flister MJ, Bivens CM et al. Nab-paclitaxel efficacy in the orthotopic model of human breast cancer is significantly enhanced by concurrent anti-vascular endothelial growth factor A therapy. Neoplasia 10(6), 613-623 (2008).

4 Weiszhar Z, Czucz J, Revesz C, Rosivall L, Szebeni J, Rozsnyay Z. Complement activation by polyethoxylated pharmaceutical surfactants: Cremophor-EL, Tween-80 and Tween-20. Eur. J. Pharm. Sci. 45(4), 492-498 (2012). 
5 Salvador-Morales C, Flahaut E, Sim E, Sloan J, Green ML, Sim RB. Complement activation and protein adsorption by carbon nanotubes. Mol. Immunol. 43(3), 193-201 (2006). Hamad I, Hunter AC, Moghimi SM. Complement monitoring of Pluronic $127 \mathrm{gel}$ and micelles: Suppression of copolymer-mediated complement activation by elevated serum levels of HDL, LDL, and apolipoproteins AI and B-100. J. Control. Release 170(2), 167-174 (2013).

7 Devine DV, Wong K, Serrano K, Chonn A, Cullis PR. Liposome-complement interactions in rat serum: implications for liposome survival studies. Biochim. Biophys. Acta 1191(1), 43-51 (1994).

8 Borchard G, Kreuter J. The role of serum complement on the organ distribution of intravenously administered poly (methyl methacrylate) nanoparticles: effects of pre-coating with plasma and with serum complement. Pharm. Res. 13(7), 1055-1058 (1996).

9 Dobrovolskaia MA, Patri AK, Zheng J et al. Interaction of colloidal gold nanoparticles with human blood: effects on particle size and analysis of plasma protein binding profiles. Nanomedicine 5(2), 106-117 (2008).

10 Goldstein JL, Ho YK, Basu SK, Brown MS. Binding site on macrophages that mediates uptake and degradation of acetylated low density lipoprotein, producing massive cholesterol deposition. Proc. Natl Acad. Sci. USA 76(1), 333-337 (1979).

11 Wang G, Simberg D. Role of scavenger receptors in immune recognition and targeting of nanoparticles. Rev. Cell Biol. Mol. Med. 1, 166-189 (2015).

12 Simberg D, Park JH, Karmali PP et al. Differential proteomics analysis of the surface heterogeneity of dextran iron oxide nanoparticles and the implications for their in vivo clearance. Biomaterials 30(23-24),3926-3933 (2009).
13 Inturi S, Wang G, Chen F et al. Modulatory role of surface coating of superparamagnetic iron oxide nanoworms in complement opsonization and leukocyte uptake. ACS Nano 9(11), 10758-10768 (2015).

14 Jung CW. Surface properties of superparamagnetic iron oxide MR contrast agents: ferumoxides, ferumoxtran, ferumoxsil. Magn. Reson. Imaging 13(5), 675-691 (1995).

15 Chao Y, Makale M, Karmali PP et al. Recognition of dextran-superparamagnetic iron oxide nanoparticle conjugates (Feridex) via macrophage scavenger receptor charged domains. Bioconjug. Chem. 23(5), 1003-1009 (2012).

16 Chao Y, Karmali PP, Mukthavaram R et al. Direct recognition of superparamagnetic nanocrystals by macrophage scavenger receptor SR-AI. ACS Nano 7(5), 4289-4298 (2013).

17 Banda NK, Mehta G, Chao Y et al. Mechanisms of complement activation by dextran-coated superparamagnetic iron oxide (SPIO) nanoworms in mouse versus human serum. Part. Fibre Toxicol. 11(1), 64 (2014).

18 Wang G, Inturi S, Serkova NJ et al. High-relaxivity superparamagnetic iron oxide nanoworms with decreased immune recognition and long-circulating properties. ACS Nano 8(12), 12437-12449 (2014).

19 Pedersen MB, Zhou X, Larsen EK et al. Curvature of synthetic and natural surfaces is an important target feature in classical pathway complement activation. J. Immunol. 184(4), 1931-1945 (2010).

20 Neun BW, Dobrovolskaia MA. Qualitative analysis of total complement activation by nanoparticles. Methods Mol. Biol. 697, 237-245 (2011). 